\title{
Review Paper \\ Abortion Laws and Regulations in Iran and European Countries During the COVID-19 Pandemic
}

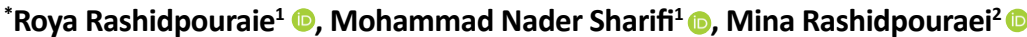

1. Department of Medical Ethics, School of Medicine, Tehran University of Medical Sciences, Tehran, Iran

2. Department of Ultrasound, The Burwin Institute of Diagnostic Medical Ultrasound, Toronto, Canada.

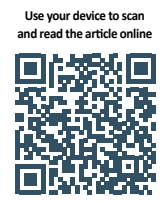

Cittation: Rashidpouraie R, Sharifi MN, Rashidpouraei M. [Abortion Laws and Regulations in Iran and European Countries During the COVID-19 Pandemic (Persian)]. Journal of Arak University of Medical Sciences (JAMS). 2020; 23(5):686-697. https://doi.org/10.32598/JAMS.23.COV.6394.1

doi https://doi.org/10.32598/JAMS.23.COV.6394.1

Key words:

Abortion, COVID-19, Illegal abortion, Induced abortion, Legal abortion

\begin{abstract}
Background and Aim Abortion has always posed challenges in the areas of ethics, law, religion, philosophy, and reproductive health. Some countries have had different approaches to abortion at different times. Today, abortion is a major challenge in Iran. Social developments and increasing level of health literacy, awareness and participation of women in social and economic fields have led to incompatibility of theoretical and practical aspects in these areas. Women sometimes have abortions without attention to the national law. In Iran, the reasons for legal abortion are the life-threatening conditions of the mother and the fetus. During the Coronavirus Disease 2019 (COVID-19) pandemic, some countries have revised their abortion laws and regulations. In some countries, such as France and Finland, these revisions are permanent; in countries such Portugal and Norway, the changes are temporary; and in other countries such as Germany and Belgium, it is unclear whether the changes are permanent or temporary. In this study, we aim to review the abortion laws and regulations in Europe and Iran. Then, by discussing the new guidelines for the COVID-19 pandemic, we evaluate the effects and consequences of this pandemic on abortion.

Methods \& Materials The search was conducted in PubMed/MEDLINE, ScienceDirect, Scopus and Google Scholar as well as national databases such as SID, MagIran, and IranMedex on studies published from 2002 to 2020 using the following keywords:Abortion, illegal abortion, induced abortion, and COVID-19. Due to the onset of SARS-COV1 epidemic in 2002, all articles published between these two outbreaks were searched. Ethical Considerations All ethical principles were observed in this article.

Results During the COVID-19 pandemic, some European countries revised the abortion laws and regulations, mostly due to realize the reproductive health right. It seems that, this revision has not yet been taken place in Iran.

Conclusion With the emergence of COVID-19, some countries had revised their abortion laws and regulations to reduce unsafe abortions. It seems that during the Covid19 pandemic, due to changes in lifestyle such as social distancing and economic and social lockdowns, it needs to revise medical laws and regulations in health-oriented and time-dependent areas (such as abortion) so that the principles of medical ethics such as beneficence and maleficent, can be applied. Having COVID-19 and consequently abortion during the pandemic is one of the most challenging issues that should be addressed in terms of ethical, jurisprudential and legal aspects. Development of regulations based on ethical principles during the $\mathrm{CO}$ VID-19 pandemic is necessary to prevent illegal and unsafe abortions.
\end{abstract}

\section{Corresponding Author:}

Roya Rashidpouraie, MD.

Address: Department of Medical Ethics, School of Medicine, Tehran University of Medical Sciences, Tehran, Iran.

Tel: +98 (912) 2002539

E-mail: dr.roya9@gmail.com 


\section{Extended Abstract}

\section{Introduction}

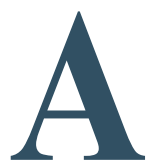

bortion has always posed challenges in the areas of ethics, law, religion, philosophy, and reproductive health. Today, abortion is a major challenge in Iran. Social developments and increasing the level of health literacy, and awareness and participation of women in social and economic fields have led to incompatibility of theoretical and practical aspects in these areas. Women sometimes have abortions without attention to sharia rules and criminal laws. In Iran, the reasons for legal abortion are the life-threatening conditions of the mother and the fetus. During the Coronavirus Disease 2019 (COVID-19) pandemic, some countries have revised their abortion laws and regulations. In some countries, such as France and Finland, these revisions are permanent; in countries such as Portugal and Norway, the changes are temporary; and in other countries such as Germany and Belgium, it is unclear whether the changes are permanent or temporary. In this study, we aim to review these revisions in Europe and Iran. Then, by discussing the new guidelines, we evaluate the effects and consequences of the COVID-19 pandemic on abortion.

\section{Material and Methods}

The search was conducted in PubMed/MEDLINE, ScienceDirect, Scopus and Google Scholar as well as national databases including SID, MagIran, and IranMedex on studies published from 2002 to 2020 using the following keywords:Abortion, induced abortion, illegal abortion, and COVID-19. Due to the onset of SARS-COV1 epidemic in 2002, all articles that had been published during these two outbreaks were searched.

\section{Results}

During the COVID-19 pandemic, some European countries revised abortion laws and regulations, mostly, due to realize the reproductive health right. It seems that this revision has not yet been taken place in Iran. By examining temporary or permanent revisions of abortion laws and regulations made in Europe, it seems that the policymakers in the field of public health in Iran during the pandemic should have a comprehensive, timely and scientific view of the effects and consequences of such diseases, because the speed of decision making and presenting policy packages, especially in cases such as abortion which is very time-sensitive, can prevent short-term and long-term complications and morbidity in pregnant women and reduce the huge economic and social costs of families and governments. Cur- rently, the guidelines of the National Coronavirus Combat and Prevention Headquarters in Iran are binding on all executive organs, and it is one of organizations that can issue instructions for temporary revision of medical abortion's regulations. Therefore, considering the high number of illegal abortions in Iran and the social restriction made by the National Coronavirus Combat and Prevention Headquarters, there is a need to issue instructions based on the principles of medical ethics.

\section{Discussion and Conclusion}

With the emergence of COVID-19, some countries had revised their abortion laws and regulations to reduce unsafe abortions. It seems that during the Covid19 pandemic, due to changes in lifestyle such as social distancing and economic and social lockdowns, there seems to be a need to revise medical laws and regulations in health-oriented and time-dependent areas (such as abortion) so that the principles of medical ethics such as beneficence and maleficent, can be applied. Having COVID-19 and consequently abortion during the pandemic is one of the most challenging issues that should be addressed in terms of ethical, jurisprudential and legal aspects. Development of regulations based on ethical principles during the COVID-19 pandemic is necessary to prevent illegal and unsafe abortions.

\section{Ethical Considerations}

\section{Compliance with ethical guidelines}

All ethical principles were observed in writing this article.

\section{Funding}

This research did not receive any specific grant from funding agencies in the public, commercial, or not-forprofit sectors.

\section{Authors' contributions}

All authors contributed equally to this work.

\section{Conflicts of interest}

The authors declare no conflict of interests.

\section{Acknowledgements}

The authors would like to thank Prof. Eskandar Omidinia for his corporation. 



\title{
بررسى قوانين و مقررات سقط جنين در ايران و ارويا در همه تيرى كوويد 19
}

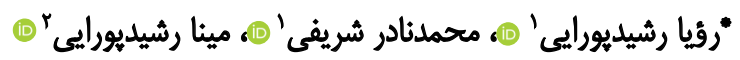 \\ ا. ا.تروه اخلاق يزشكى، دانشكده يزشكى، دانشكاه علوميزشكى تهران، تهران، ايران.

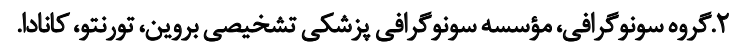

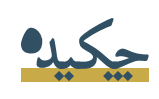

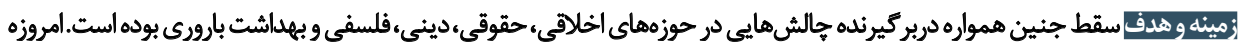

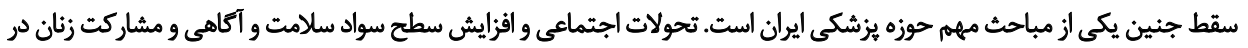

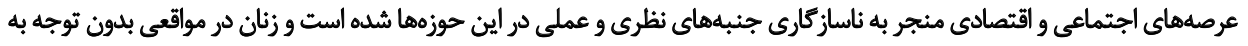

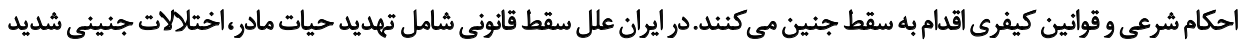

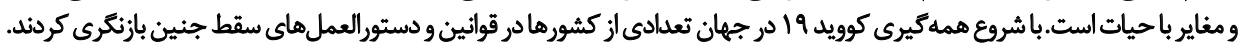

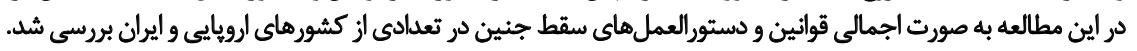

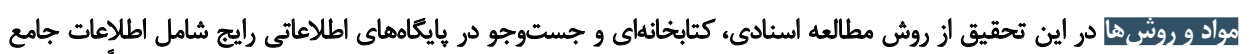

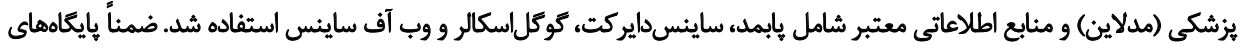

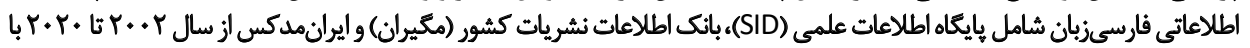

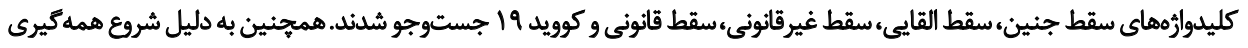

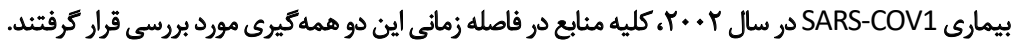

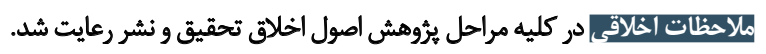

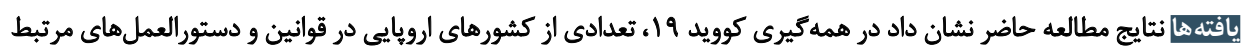

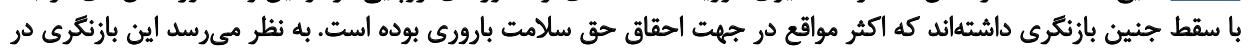

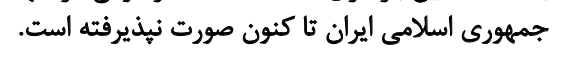

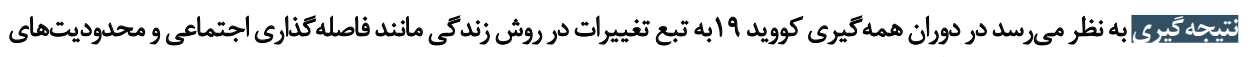

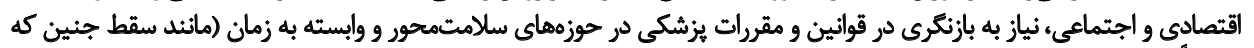

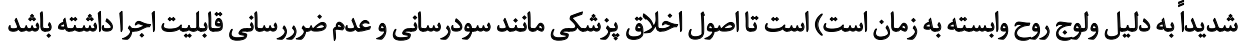
واز سقطهاي غيرايمن بيشكيرى شود.
\end{abstract}

اطلاعات مقاله:

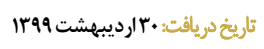

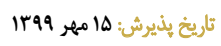

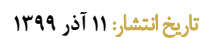

كليدوازوها:

سقط القايي، سقط جنين، سقط قانونى، سقو سقط غيرقانوني، كوويد

19

حوزهها شده است و زنان در مواقعى، بدون توجه به احكام شرعى إئ

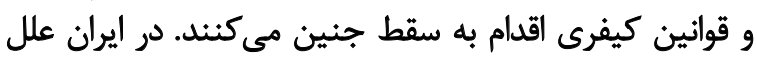

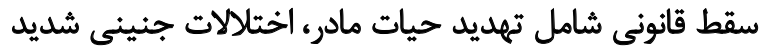

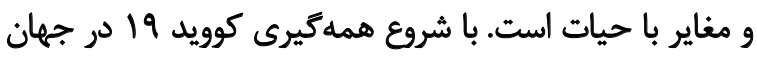

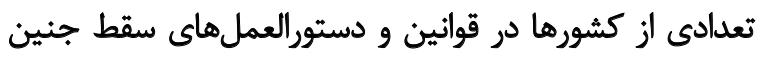

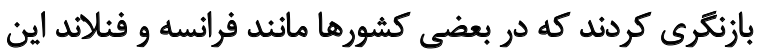

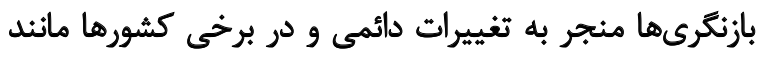

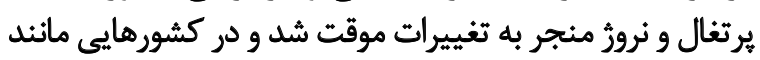

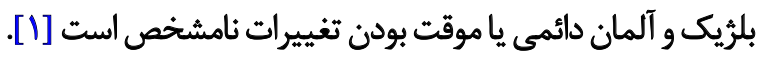

سقط جنين همواره دربركيرنده جالشهايى در حوزههاي

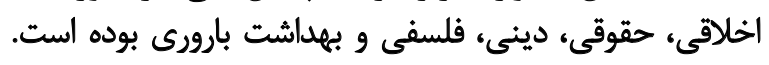

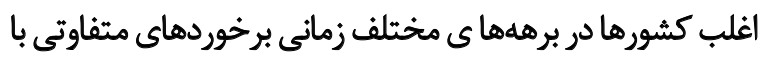

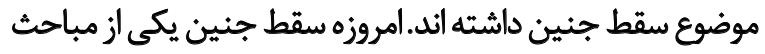

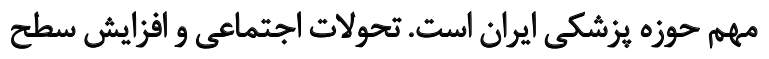

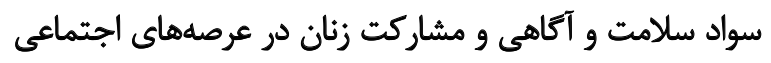

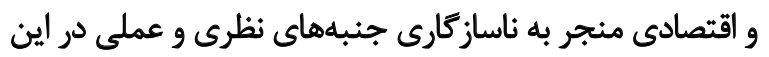


عنوان يكى از خدمات سلامت در طول همهكيرى كوويد 19 در

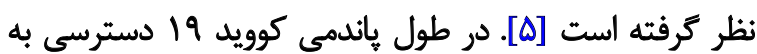

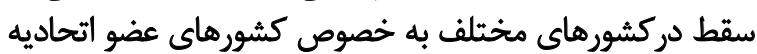

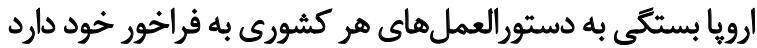

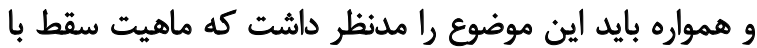

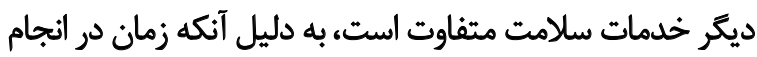

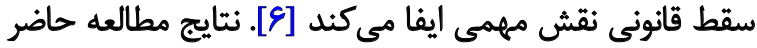

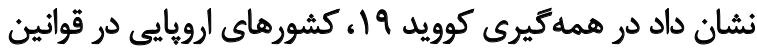

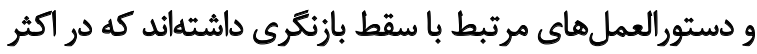

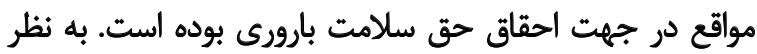

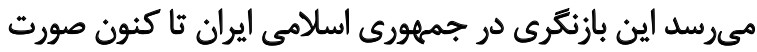

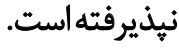
در اين مقاله ابتدا به مفاهيم سقط جنين يرداخته و سيس قوانين سقط جنين در دوران قبل و بعد از همه جيرى كورين

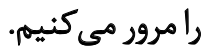

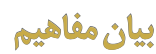

تعر يف بسقط جنين

سقط جنين را مىتوان از ديدكاه يزشكى، فقهى و جزايى

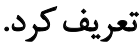

\section{تعريف سقط جثين در اصطلاح برُشكى}

سقط جنين از نظر يزشكى عبارت است از اخراج عمدى يا يا

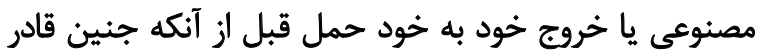

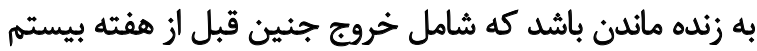

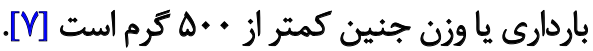

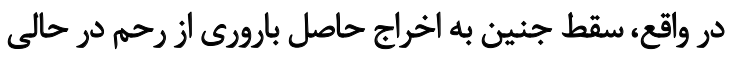

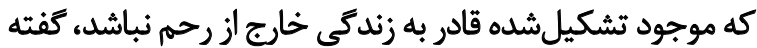

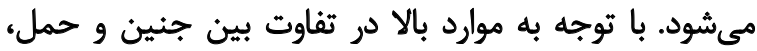

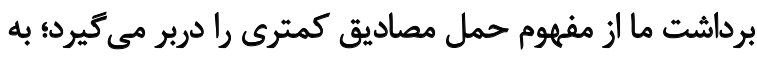

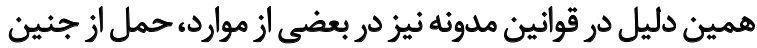

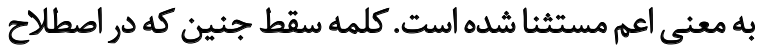

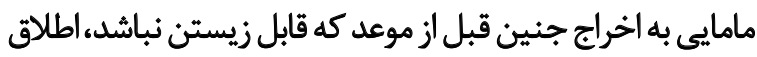

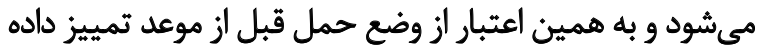

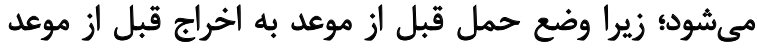

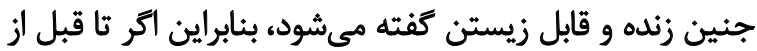

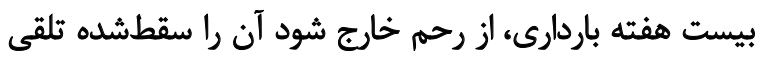

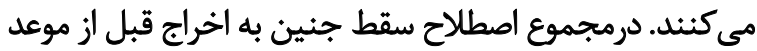
جنين به نحوى كه قابل زيستن نباشد، اطلاق مى شيود. تعريف سقط جثين در اصطلاح فقهى موارد تعريف سقط جنين در مدارك فقهى بسيار نادر است. اغلب
كوويد 19 و به تبع آن، سقط در دوران همهكيرى يكى از جازئ

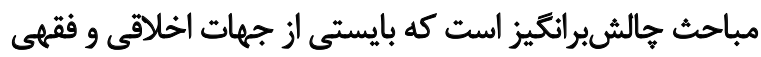

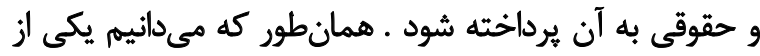

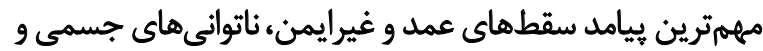

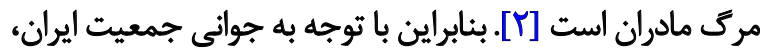

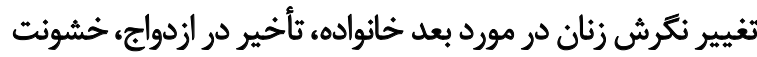

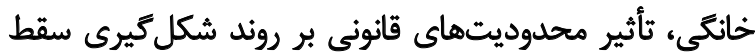

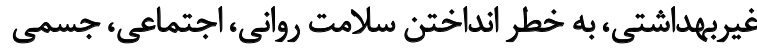

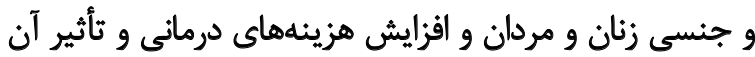

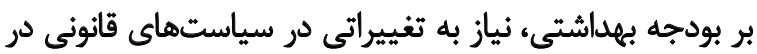

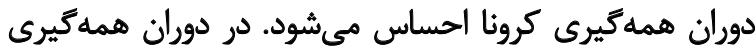

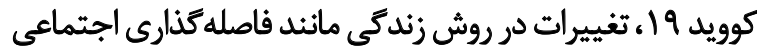

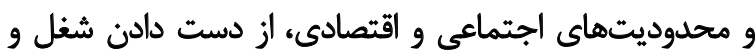

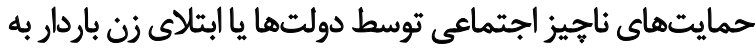

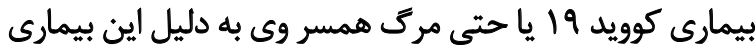

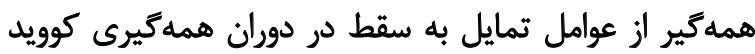

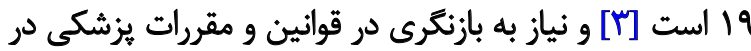

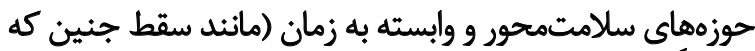

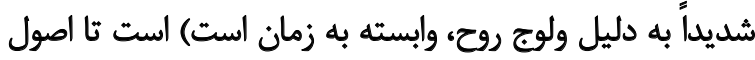

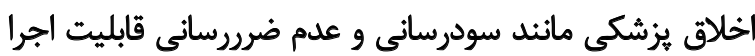

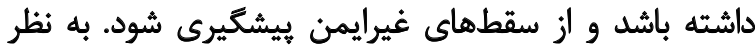

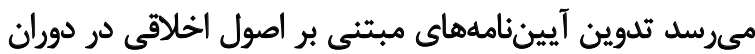

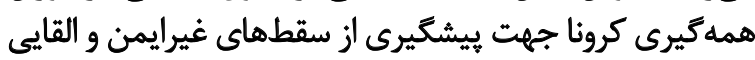

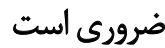

مواد و روشُ نها

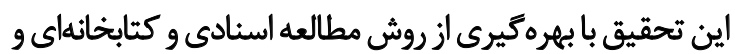

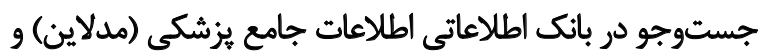

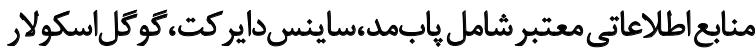

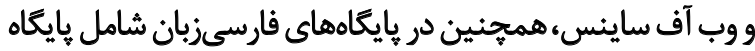

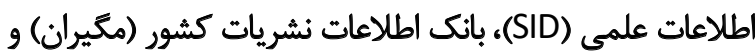

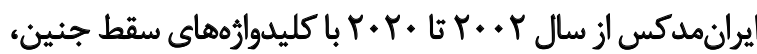

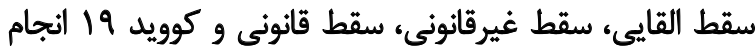

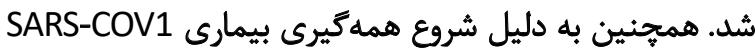

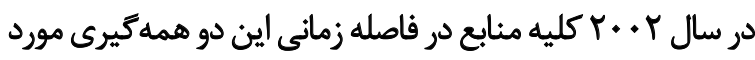

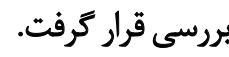

ياقتهها

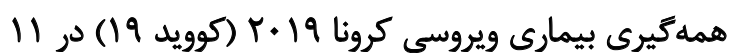

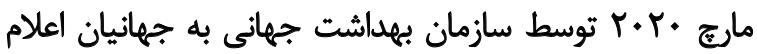

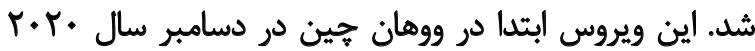

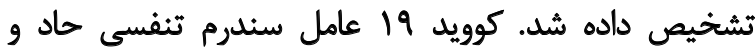

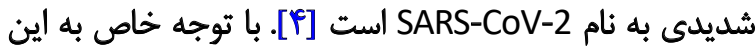

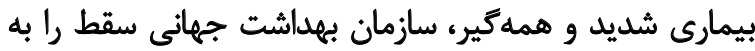


موارد ثجويزٌ سيقط جثين

اتر حه در اغلب نظامهاى حقوقى اقدام به سقط جنين عملى

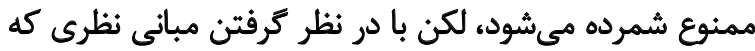

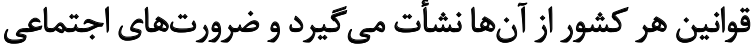

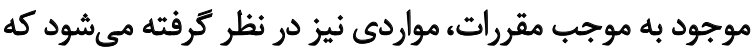

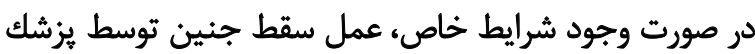

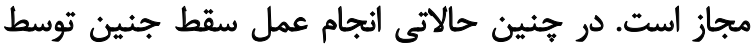
يزشك بايستى با تشريفات خاص صورت كيرد.

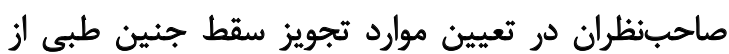

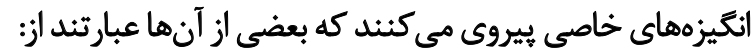

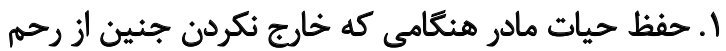
وى يا وضع حمل، آن را به خطر بيندازد.

r. جلوكيرى از تولد نوزاد ناقصالخلقه و معلول كه داراى

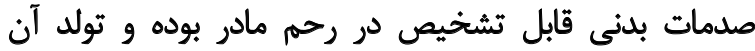

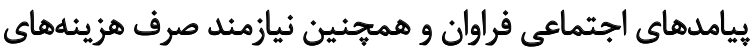
هنكفت براى نتخهدارى است.

". بيشكيرى از ضررهاى معنوى و حفظ آبرو كه در بى آشكار

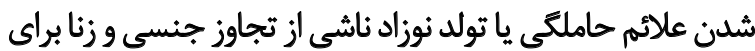
زنان و دختران حامله ايجاد خواهد شاند.

موارد تجويز سقط جثين توسط ئرشك در قواثين جارى (فعلى) طبق مصوبه مجلس شوراى اسلامى، سقط جنين بر اساس ماده واحده زير جايز است:

يزاسكطدرمانى با تشخيص قطعى سه بزشك متخصص و وتأييد

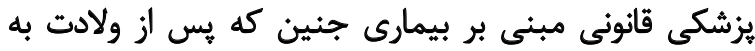

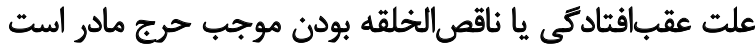

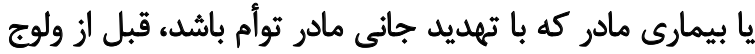

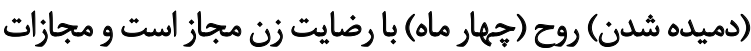

و مسئوليتي متوجه هيزشك مباشر نخواهد بوده) [ • (1]. بنابراين بر اساس اين قانون، سقط جنين در موارد غير از آنجهه

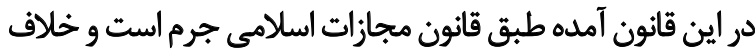
شرع محسوب مي شون امدهو.

\section{وضعيت سمط جثين در جهان}

سقط جنين در سطح جهان رو به افزايش است كه به دلايلى

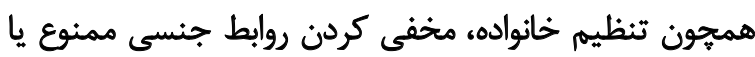

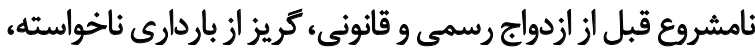

هتك ناموس به عنف و غيره واقع مى ازشود.

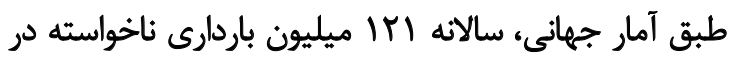

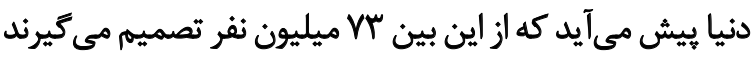

فقها در ورود به بحث ديه جنين به ذكر حالات مختلف آن در بطن

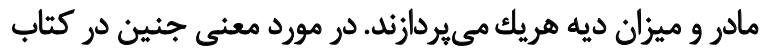

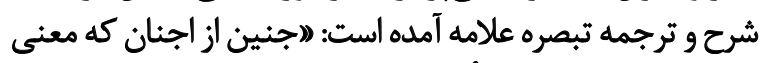
آن مستور بودن است، مأخوذ است (مستور در رحم)ه.

همجنين شهيد ثانى در شرح لمعه در باب شرح و توضيح كلمه

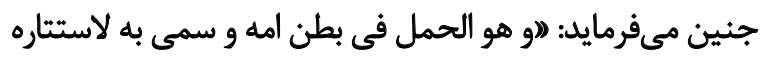
فيه من الاجتنان و هوالستر فهو بمعنى المفعول؛ يعنى جني جنين

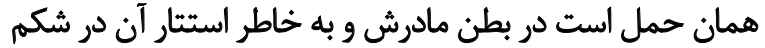

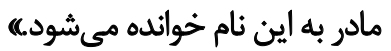

در متون فقهى اسلام با استفاده از آيات قرآنى و روايات وارده إنه

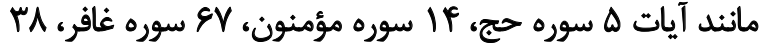

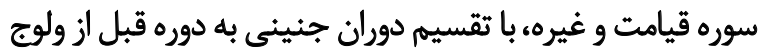

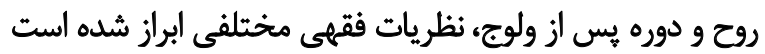

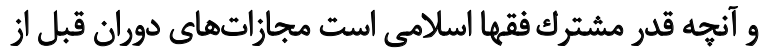

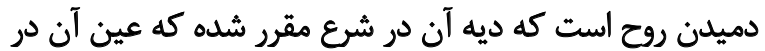

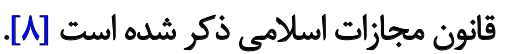

تعريف سقط جنين در اصطاح جزايف

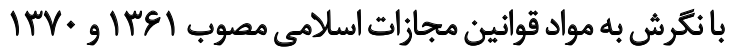

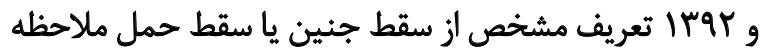

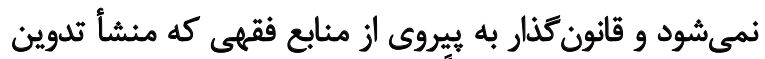

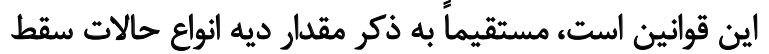

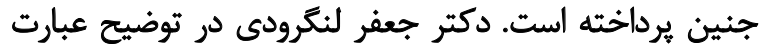

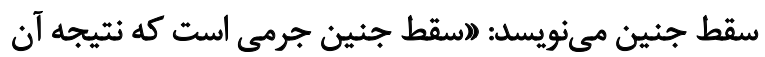

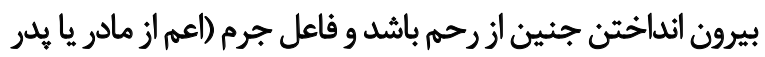

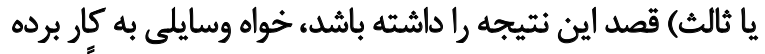

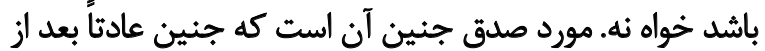

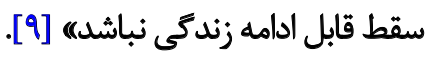

در مواردى كه سقط را قانون تجويز كنده جرم سقط صدق ديق

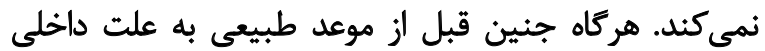

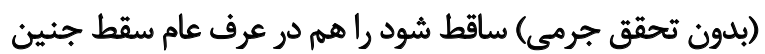

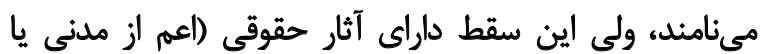

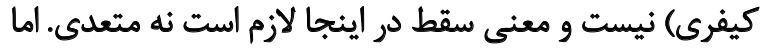

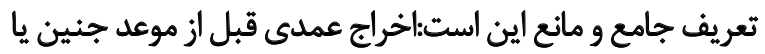

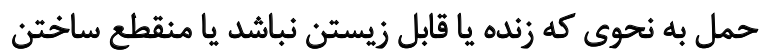

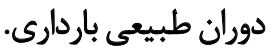

به هر حال در مقايسه دو تعريف يزشكى و حقوقى سقط جنين.

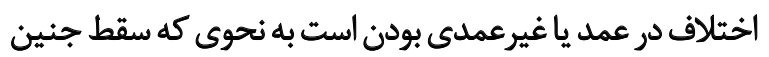

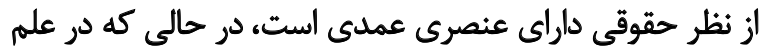
يزشكى عدم شرطيت عنصر عمد وجود دارد. 
يافته است، در حالى كه در كشورهاى در حال توسعه در همان

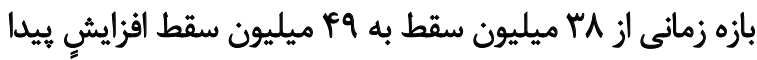

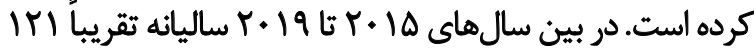

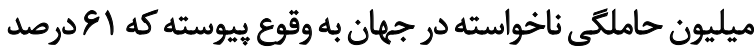

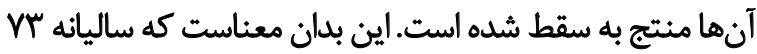

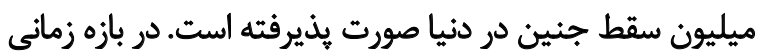

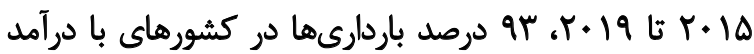

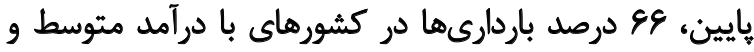
FF

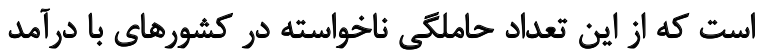

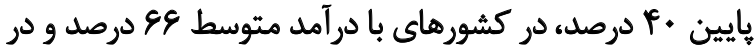

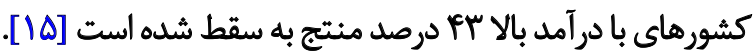

سقط جثين در اليران

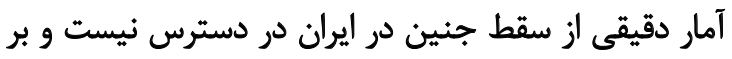

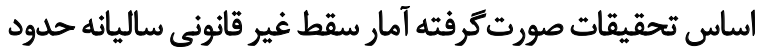

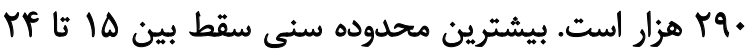

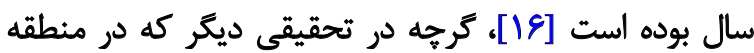

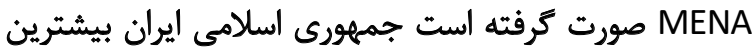

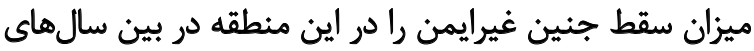

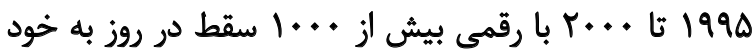

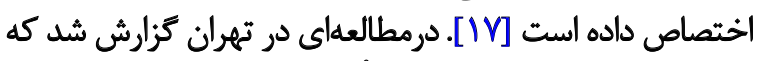

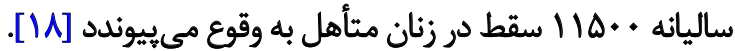

\section{قواثين سقط جثين در كشورهاى اروياييى}

سقط جنين يكى از اقدامات شايع در زنان در سنين بارورى

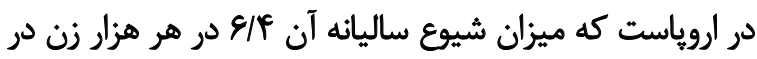

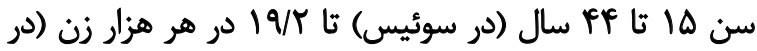

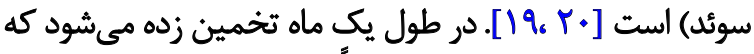

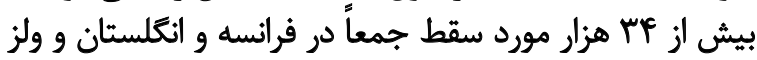

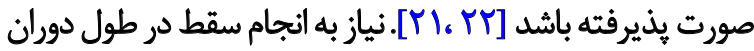

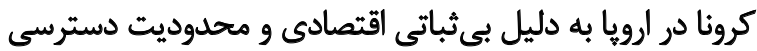

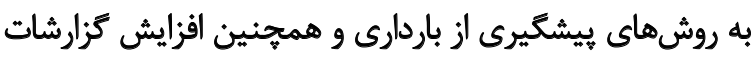

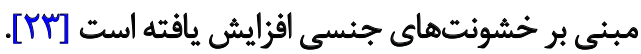

در همدكيرى كرونا كشورهاى ارويايى رويكردهاى متفاوتى را

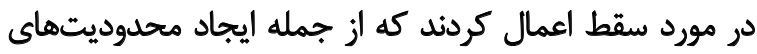

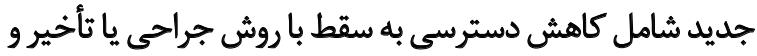

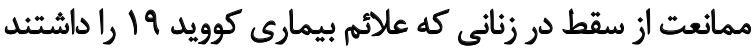

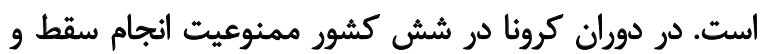

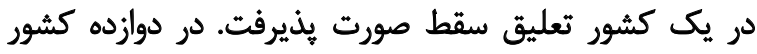

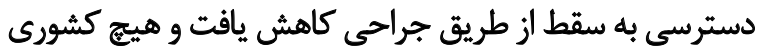

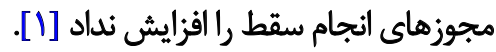

مادر نشوند و اقدام به سقط جنين خود مي كنند. طبق اين آمار،

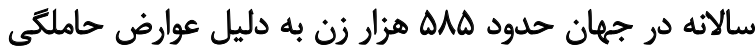

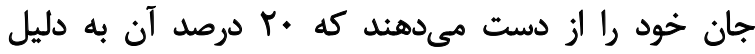

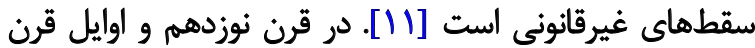

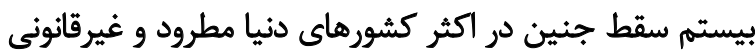

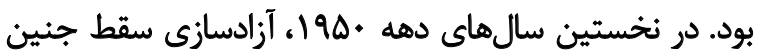

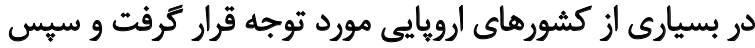

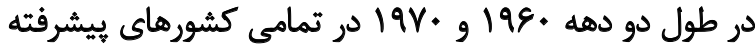

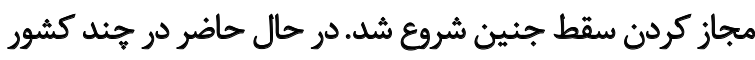

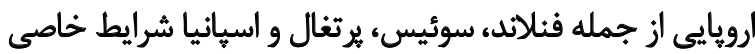

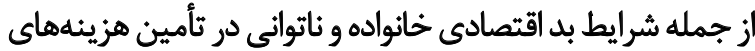

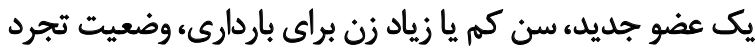

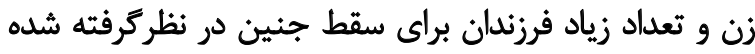

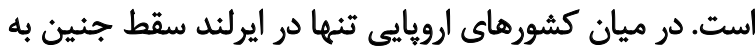

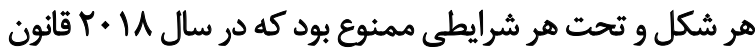

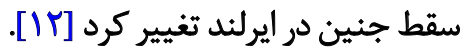
در سال 1999 ميلادى سازمان ملل متحد در يك ئروهش

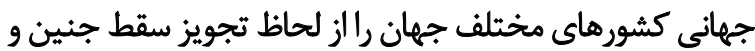

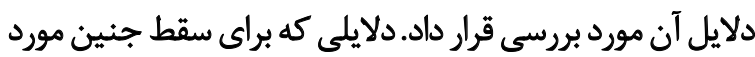

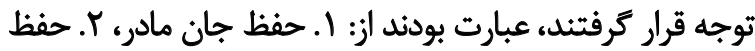

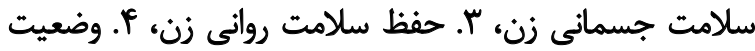

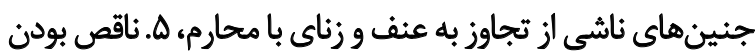

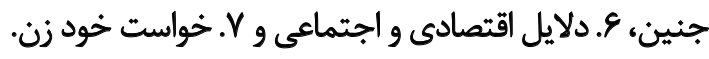
مطابق اين تحقيق از ميان بوان اكشور جهان، 199 اكشور سقط جنين رابراي حفظ جان مادر مجاز كردهاند. سقط جنين إنين به دليل

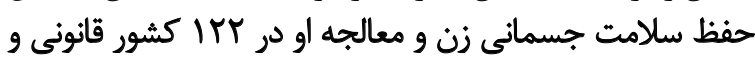

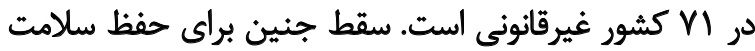

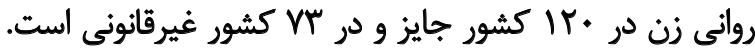

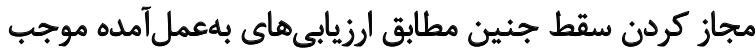

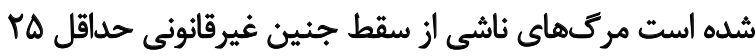

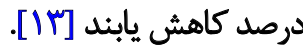

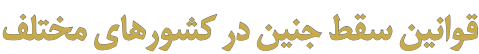

\section{سقط جثين از ديد كاه جهاني}

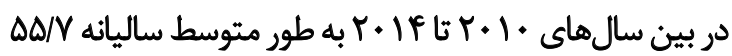

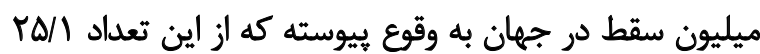

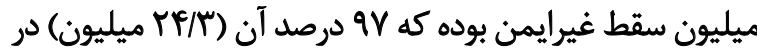

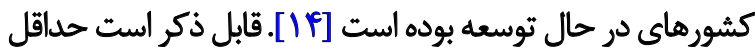

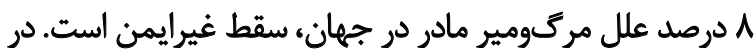

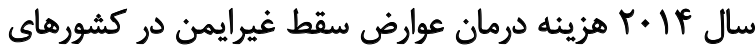

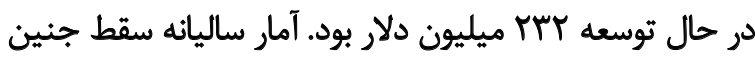

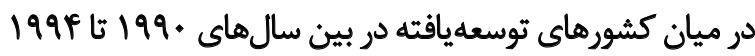

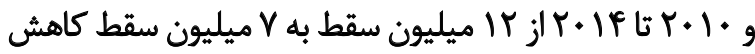




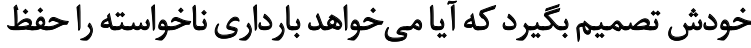

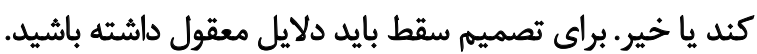

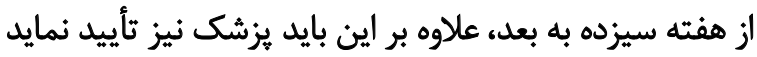

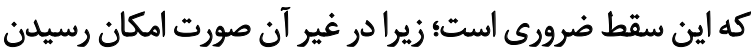

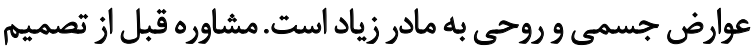

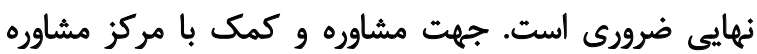

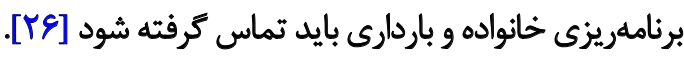

دائمارى

در دانمارك، زنان اين حق را دارند كه در دوازده هفته اول

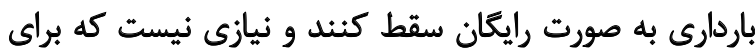

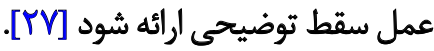

ايثاليا

در سال 19 اسقط در سه ماهه اول باردارى و تنهادر صورتى

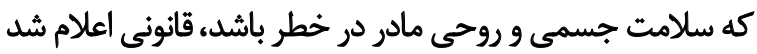

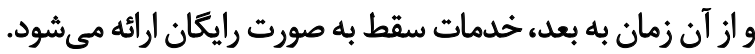

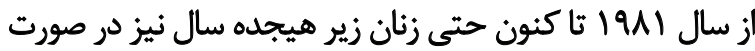

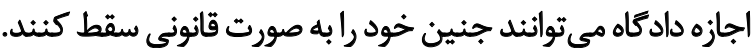

$$
\text { (آلبانيز، + + . r ميلادى). }
$$

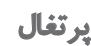

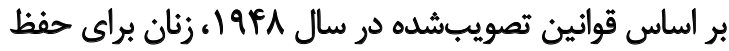

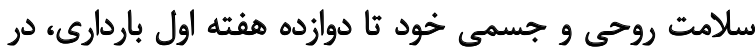

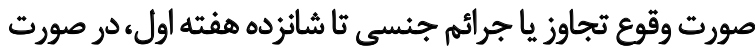

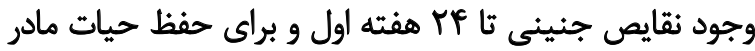

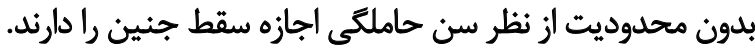

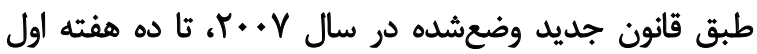

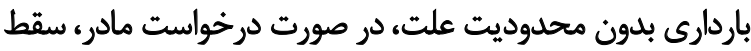
قانونى شمرده مي شود (فدراسيون بينالمللى، 9 ×. ب ميلادى).

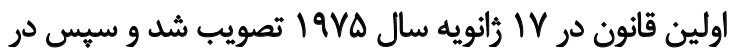

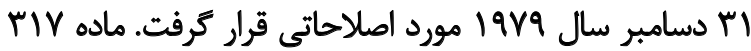

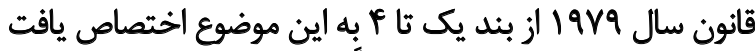

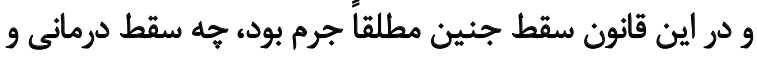

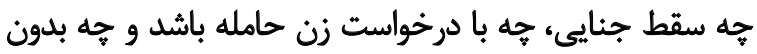

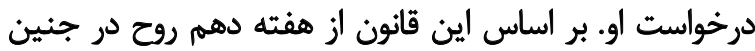

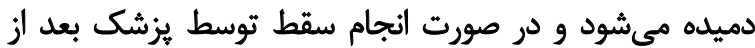

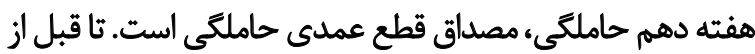

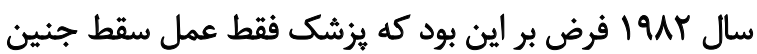

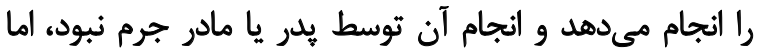

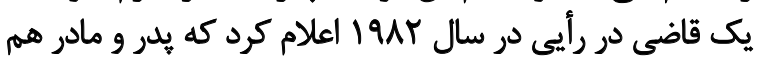

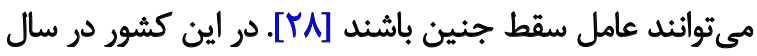

در آلمان، تحت شرايط خاصى مىتوان اقدام به سقط جنين

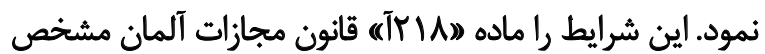

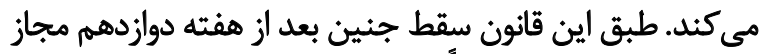

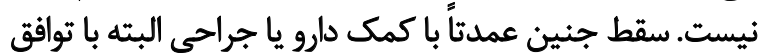

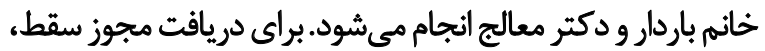

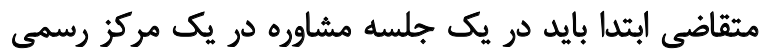

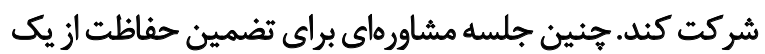

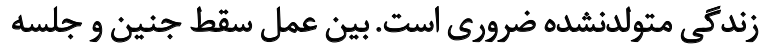
مشاوره حداقل بايد سه روز فاصله وجود داشته باشند.

اتر سقط جنين قرار است به دليل مشكلات يزشكى انجام

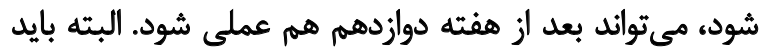

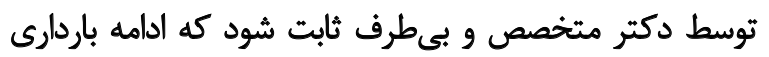

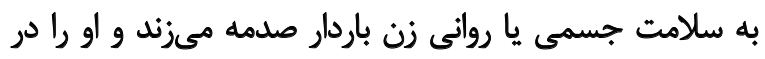

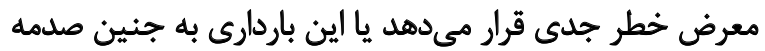

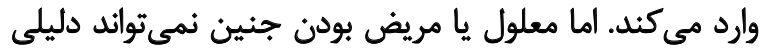

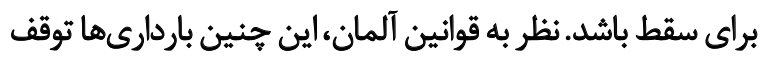

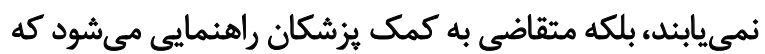

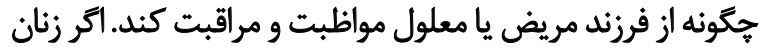

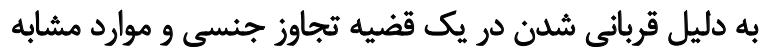

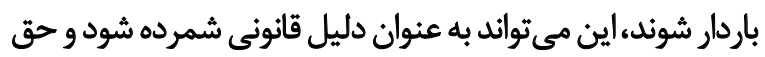

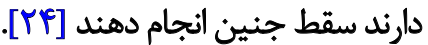

سوئ

آخرين قانون سقط جنين در سوئد مصوب سال I9VF است

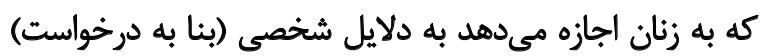

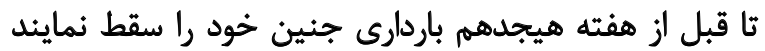

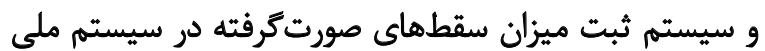

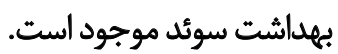

39

سقط جنين تا هفته هيجدهم باردارى به درخواست زن باردار مجاز است.

فنلاثد

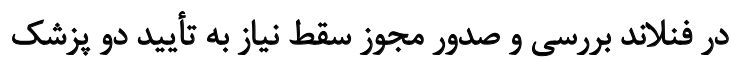

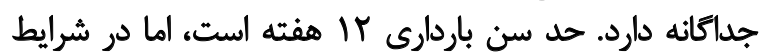

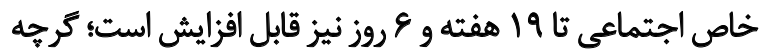

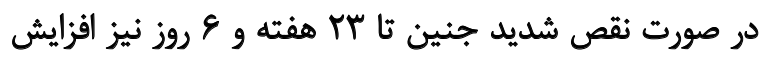

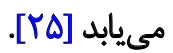

سوئيس

ختم باردارى (سقط جنين) در سوئيس تحت شرايط خاصى،

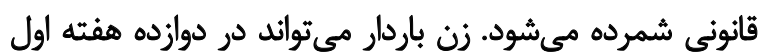


با بررسى بازنترى بهاى موقت يا دائمى در قوانين سقط جنين

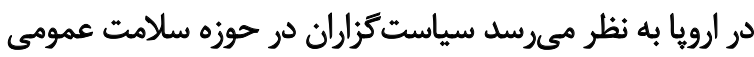

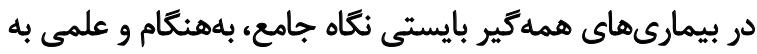

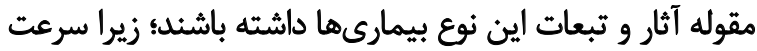

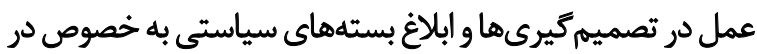

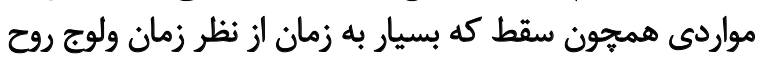

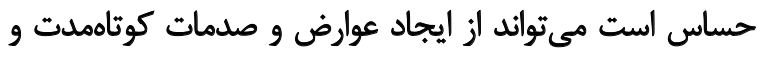

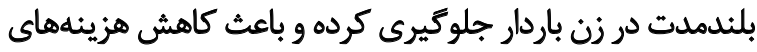

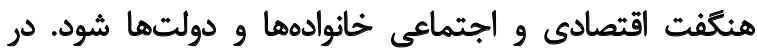

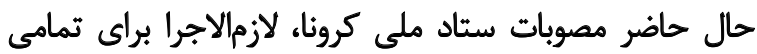

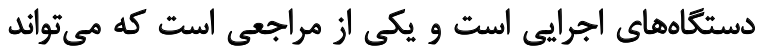

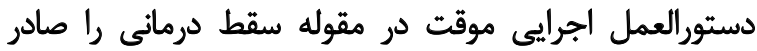

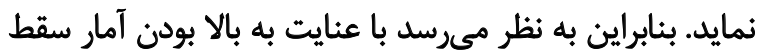

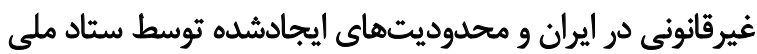

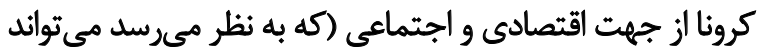

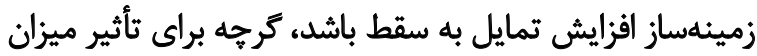

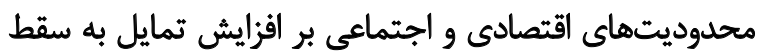

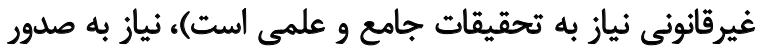

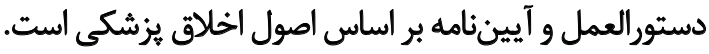

\section{نتيجليَيرى}

با شروع همدكيرى كوويد 19 در جهان تعدادى از كشورها

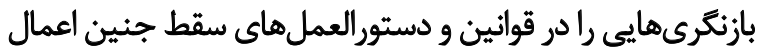

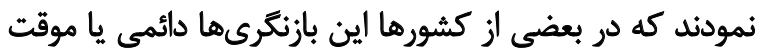

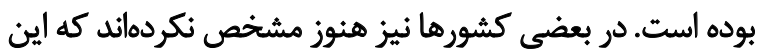
بازنكرى هاى موقت يا دائمى خواهد شد شد.

سقط جنين از جمله مسايل فراكير در جوامع بشرى است كه نهنتئها

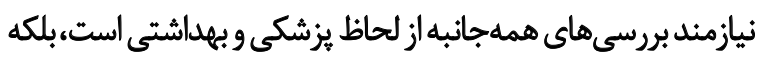

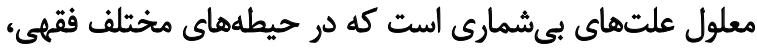

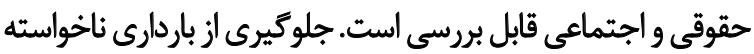

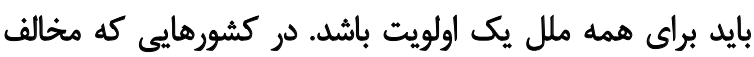

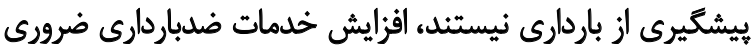

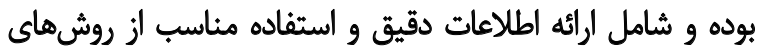

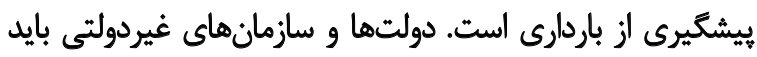

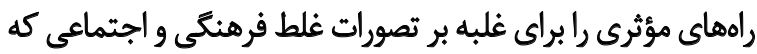

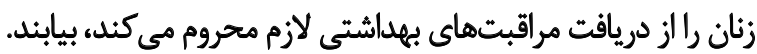

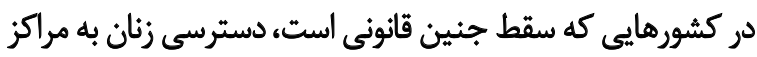

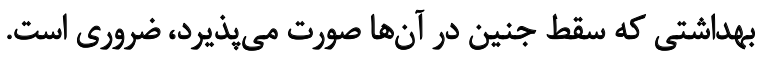

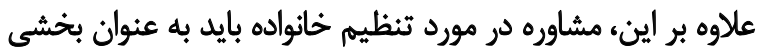

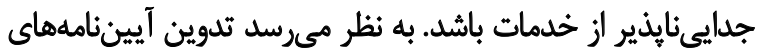

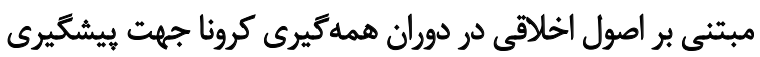
از سقطهاى غيرايمن و القايى ضرورى است.

در تدوين آيينامههاى مرتبط باسقط جنين همواره بايداتونومى،

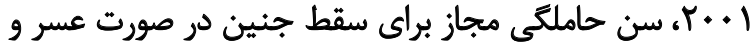

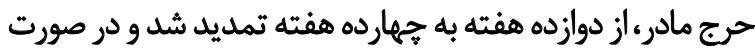

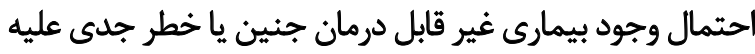

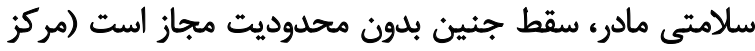

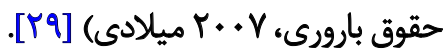

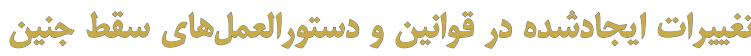

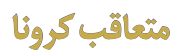

بلزيك: در تعدادى از بيمارستانها دسترسى به سقط محدود

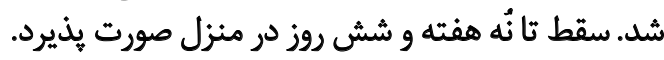

فرانسه: اجازه سقط در منزل از هفت هفته بها نُه هفته افزايش

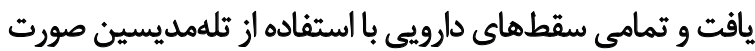

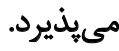

فنلاند: اجازه سقط در منزل از نُه هفته به ده هفته افزايش هافت.

آلمان: مشاوره جهت انجام سقط جنين از طريق تلهمديسين اجبارى شد و بيشتر سقطها به صورت جراحى صورت مى ليذيرد.

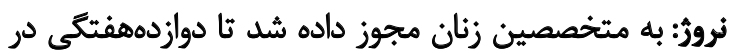
خارج از بيمارستان بتواند سقط رانجام انجام دهند.

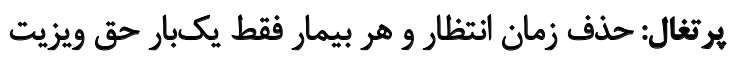
يزشك براي انجام سونوكرافى و سقط دارد و فالوآب بايستى توسط تلممديسين صورت بذيرد.

سوئيس: الجازه سفط توسط دارو از هفت هفته به نه هفته [فزايش يافت [1].

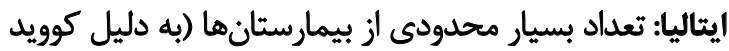

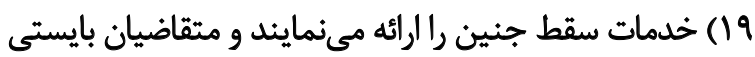

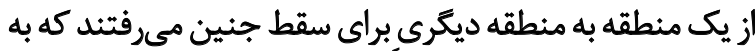

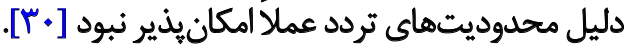

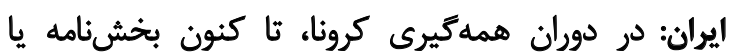
درستورالعملى در خصوص بازنكرى يا تغيير در اجراى قانون سقط درمانى صادر نشده است.

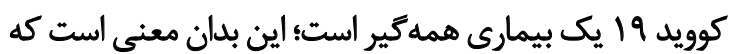

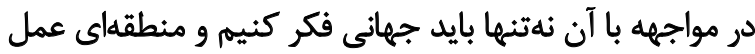
كنيم، بلكه بايد اصول اخلاق يزشكى را هم در نظر داشته بائه باشيم

با شروع همهكيرى كوويد 19 در جهان بسيارى از كشورها

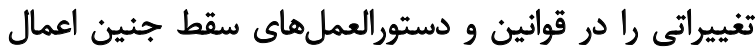

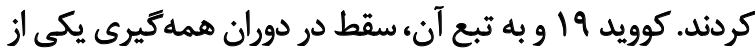
مباحث جالشبرانكيز است كه بايستى از جهات اخلافى و فقيهى و 9 حقوقى به آن يرداخته شود. 
حق رعايت رازداري، ارزش كذارى به زمينههاي فكرى و فرهنكى و و

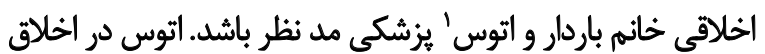

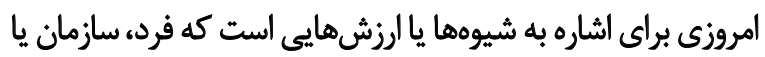

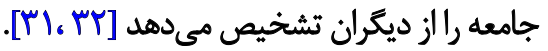
مالاحظات اخلاقى - اتى

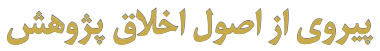

با توجه به اينكه در اين مطالعه از نمونه انسانى يا جانورى

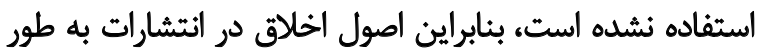
كامل رعايت شده است.

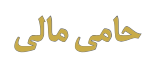
اين تحقيق هيج كونه كمك مالى از سازمان هاى تأمين مالى در بخش هاى عمومى ، تجارى يا غير انتفاعى دريافت نكرد.

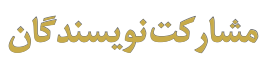

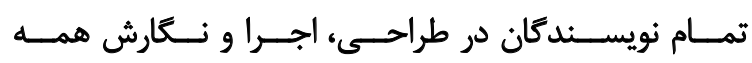

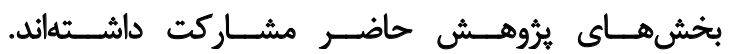

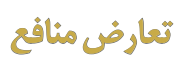

بنابر اظهار نويسندكان اين مقاله تعارض منافع ندارد.

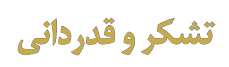

از استاد ارجمند جناب آقاى دكتر اسكندر اميدىنيا كمال تشكر و قدردانى را داريم. 


\section{References}

[1] Moreau C, Shankar M, Glasier A, Cameron S, Gemzell-Danielsson K. Abortion regulation in Europe in the era of COVID-19: A spectrum of policy responses. BMJ Sexual \& Reproductive Health. 2020; bmjsrh-2020-200724. [DOI:10.1136/bmjsrh-2020-200724] [PMID]

[2] Latt SM, Milner A, Kavanagh A. Abortion laws reform may reduce maternal mortality: An ecological study in 162 countries. BMC Women's Health. 2019; 19(1):1. [DOI:10.1186/s12905-0180705-y] [PMID] [PMCID]

[3] Rashidpouraie R, Sharifi MN. Covid19 and abortion right, Obstet Gynecol Sci. 2020; 63(6):743-4. [DOI:10.5468/ogs.20185] [PMID] [PMCID]

[4] World Health Organization (WHO). WHO Timeline - COVID-19 [Internet]. 2020 [Updated 2020 June 29]. Available from: https:// www.who.int/news/item/27-04-2020-who-timeline---covid-19

[5] World Health Organization (WHO). COVID-19:Operational guidance for maintaining essential health services during an outbreak:Interim guidance [Internet]. 2020 [Updated 2020 March 25]. Available from: https://apps.who.int/iris/handle/10665/331561

[6] Hussein J. COVID-19: What implications for sexual and reproductive health and rights globally? Sex Reprod Health Matters. 2020; 28(1):1746065 [DOI:10.1080/26410397.2020.1746065] [PMID]

[7] Cunningham F, Leveno K, Bloom S, Spong CY, Dashe J. Williams obstetrics, 25e. New York: Mcgraw-Hill; 2018.

[8] Mosavi Bojnordi SM. [A jurisprudential and legal study of abortion with an approach to the theories of Imam Khomeini (Persian)]. Pajoheshnameh Matin. 2005; (28-29):41-56. http:// ensani.ir/file/download/article/20110131161912-

[9] Pad E. [Exclusive criminal law, Crimes against persons (Persian)]. Tehran: University of Tehran Press; 1968.

[10] Larijani B, Zahedi F. Changing parameters for abortion in Iran. Indian J Med Ethics. 2006; 3(4):130-1.

[11] Bearak J, Popinchalk A, Ganatra B, Moller AB, Tunçalp Ö, Beavin $C$, et al. Unintended pregnancy and abortion by income, region, and the legal status of abortion: Estimates from a comprehensive model for 1990-2019. The Lancet. 2020; 8(9):e1152-e1e61 [DOI:10.1016/S2214-109X(20)30315-6]

[12] Fischer C. Abortion and reproduction in Ireland: Shame, nationbuilding and the affective politics of place. Feminist Review. 2019; 122(1):32-48. [DOI:10.1177/0141778919850003]

[13] Lavelanet AF, Schlitt S, Johnson BR, Ganatra B. Global Abortion Policies Database: $A$ descriptive analysis of the legal categories of lawful abortion. BMC Int Health Hum Rights. 2018; 18(1):18. https://doi.org/10.1186/s12914-018-0183-1

[14] Ganatra B, Gerdts C, Rossier C, Ronald Johnson Jr B, Tunçalp Ö, Assifi A, et al. Global, regional, and subregional classification of abortions by safety, 2010-14:Estimates from a Bayesian hierarchical model. Lancet. 2017; 390(10110):2372-81 .[DOI:10.1016/ S0140-6736(17)31794-4]

[15] Guttmacher Institute. Unintended pregnancy and abortion worldwide [Internet]. 2020. [Updated 2020 July]. Available from: https://www.guttmacher.org/fact-sheet/induced-abortionworldwide\#
[16] Rastegari A, Baneshi MR, Haji-Maghsoudi S, Nakhaee N, Eslami M, Malekafzali $\mathrm{H}$, et al. Estimating the annual incidence of abortions in Iran applying a network scale-up approach. Iran Red Crescent Med J. 2014; 16(10):e15765 [DOI:10.5812/ircmj.15765]

[17] Hessini L. Abortion and Islam:Policies and practice in the Middle East and North Africa. Reprod Health Matters. 2007; 15(29):7584. [DOI:10.1016/S0968-8080(06)29279-6]

[18] Erfani A. Induced abortion in Tehran, Iran: Estimated rates and correlates. Int Perspect Sex Reprod Health. 2011; 37(3):134-42 [DOI:10.1363/3713411] [PMID]

[19] Federal Statistical Office of Switzerland. Interruptions de grossesse [Internet]. 2020 [Updated 2020 May 18]. Available from: https://www.bfs.admin.ch/bfs/fr/home/statistiques/ sante/etat-sante/reproductive/interruptions-grossesses.html

[20] National Board of Health and Welfare. Swedish Ministry of Health. Statistics on abortion in 2018 [Internet]. 2020 [Updated 2020 June 10]. Available from: https://www.socialstyrelsen. se/globalassets/sharepoint-dokument/artikelkatalog/statistik/2019-5-18.pdf

[21] Direction de la recherche, des études, de l'évaluation et des statistiques. 224300 interruptions volontaires de grossesse en 2018 (France) [Internet]. 2019 [Updated 2020 May 18]. Available from: https:// drees. solidarites- sante. gouv. fr/ IMG/ pdf/ er1125. pdf

[22] Department of Health \& Social Care. Abortion statistics, England and Wales, 2018 [Internet]. 2019 [Updated 2019 June 13]. Available from: https:// assets. publishing. service. gov. uk/ government/ uploads/ system/ uploads/ attachment_data/ file/ 808556/ Abortion Statistics England and Wales 2018 1_.pdf

[23] Roesch E, Amin A, Gupta J, García-Moreno C. Violence against women during Covid-19 pandemic restrictions. BMJ. 2020 369:m1712. [DOI:10.1136/bmj.m1712] [PMID] [PMCID]

[24] Handbookgermany. Pregnancy and child care in Germany [Internet]. 2020 [Updated 2020]. Available from: https://handbook germany.de/fa/live/pregnancy.html

[25] Tullberg BS, Lummaa V. Induced abortion ratio in modern Sweden falls with age, but rises again before menopause. Evol Human Behav. 2001; 22(1):1-10. [DOI:10.1016/S1090-5138(00)00057-X]

[26] Schweizerisches Rotes Kreuz. Swiss Health Guide 2011 [Internet]. 2011 [Updated 2011]. Available from: https://assets.ctfassets.net/fclxf7o732gj/4SSYvT6PGoCuCYKliM8ils/81a3db38baffa 13788670fd4accfbe75/far_GWW_web_1_.pdf

[27] Sundhedsvæsen. Danmark Health Care System [Internet]. 2016 [Updated 2016]. Available from: https://www.sst.dk/-/media/ Udgivelser/2017/Det-danske-sundhedsv\%C3\%A6sen/Det-Danske-Sundhedsv\%C3\%A6sen,-d-,-Farsi.ashx

[28] Rahpeik H, Shabani Kandsari H, Saatchi A. [Nature of dissolution of contract in shi'it jurisprudence and iranian law, with comparative study in England and French Law (Persian)]. Judicial Law Views Quarterly (Law Views). 2015; 20(70):35-56. http://jlviews. ujsas.ac.ir/article-1-453-fa.htm

[29] Rostami S, Abdi F, Ahmadi M, Dadahir AA. [Comparative study of abortion laws in countries around the world (Persian)]. Journal of Medical History. 2014; 17(5):79-111. [DOI:10.22037/mhj. v5i17.5797] 
[30] Cioffi A, Cioffi F, Rinaldi R. COVID-19 and abortion:The importance of guaranteeing a fundamental right. Sex Reprod Healthc. 2020; 25:100538. [DOI:10.1016/j.srhc.2020.100538] [PMID]

[31] Zahedi F, Tirgar S, Hamidi Abarghoei N, Rashidpouraei R, Larijani B. [Considering Islamic principles in ethical counseling for mothers of fetuses with congenital anomalies (Persian)]. Med Ethics History Med. 2015; 7(5):1-16. https://ijme.tums.ac.ir/article1-5472-en.html

[32] Sharifi MN, Namazi HRH, Rashidpouraie R. Ethical frameworks and human dignity in stopping the treatment of end stages patients with cancer. Journal of Science and Engineering Elites. 2020; 1(5):13-31. https://www.sid.ir/fa/Journal/ViewPaper. aspx?id=537019 